\title{
Highly directive and Gaussian far-field emission from "giant" photonic trumpets
}

Stepanov, Petr; Delga, Adrien; Gregersen, Niels; Peinke, Emanuel; Munsch, Mathieu; Teissier, Jean; Mørk, Jesper; Richard, Maxime; Bleuse, Joel; Gérard, Jean-Michel

Total number of authors:

11

Published in:

Applied Physics Letters

Link to article, DOI:

$10.1063 / 1.4932574$

Publication date:

2015

Document Version

Publisher's PDF, also known as Version of record

Link back to DTU Orbit

Citation $(A P A)$ :

Stepanov, P., Delga, A., Gregersen, N., Peinke, E., Munsch, M., Teissier, J., Mørk, J., Richard, M., Bleuse, J., Gérard, J-M., \& Claudon, J. (2015). Highly directive and Gaussian far-field emission from "giant" photonic trumpets. Applied Physics Letters, 107(14), [141106]. https://doi.org/10.1063/1.4932574

\section{General rights}

Copyright and moral rights for the publications made accessible in the public portal are retained by the authors and/or other copyright owners and it is a condition of accessing publications that users recognise and abide by the legal requirements associated with these rights.

- Users may download and print one copy of any publication from the public portal for the purpose of private study or research.

- You may not further distribute the material or use it for any profit-making activity or commercial gain

- You may freely distribute the URL identifying the publication in the public portal 


\title{
Highly directive and Gaussian far-field emission from "giant" photonic trumpets
}

\author{
Petr Stepanov, ${ }^{1,2}$ Adrien Delga, ${ }^{1,2}$ Niels Gregersen, ${ }^{3}$ Emanuel Peinke, ${ }^{1,2}$ Mathieu Munsch, ${ }^{4}$ \\ Jean Teissier, ${ }^{4}$ Jesper Mørk, ${ }^{3}$ Maxime Richard, ${ }^{1,5}$ Joël Bleuse,${ }^{1,2}$ Jean-Michel Gérard, ${ }^{1,2}$ \\ and Julien Claudon ${ }^{1,2, a)}$ \\ ${ }^{1}$ Univ. Grenoble Alpes, F-38000 Grenoble, France \\ ${ }^{2}$ CEA, INAC-SP2M, "Nanophysique et Semiconducteurs" Group, F-38000 Grenoble, France \\ ${ }^{3}$ DTU Fotonik, Department of Photonics Engineering, Technical University of Denmark, Building 343, \\ DK-2800 Kongens Lyngby, Denmark \\ ${ }^{4}$ Department of Physics, University of Basel, Klingelbergstrasse 82, CH-4056 Basel, Switzerland \\ ${ }^{5}$ CNRS, Institut Néel, "Nanophysique et Semiconducteurs" Group, F-38000 Grenoble, France
}

(Received 24 July 2015; accepted 25 September 2015; published online 6 October 2015)

\begin{abstract}
Photonic trumpets are broadband dielectric antennas that efficiently funnel the emission of a pointlike quantum emitter-such as a semiconductor quantum dot—into a Gaussian free-space beam. After describing guidelines for the taper design, we present a "giant" photonic trumpet. The device features a bottom diameter of $210 \mathrm{~nm}$ and a $5 \mu \mathrm{m}$ wide top facet. Using Fourier microscopy, we show that $95 \%$ of the emitted beam is intercepted by a modest numerical aperture of 0.35 . Furthermore, far-field measurements reveal a highly Gaussian angular profile, in agreement with the predicted overlap to a Gaussian beam $\mathcal{M}_{g}=0.98$. Future application prospects include the direct coupling of these devices to a cleaved single-mode optical fiber. The calculated transmission from the taper base to the fiber already reaches 0.59 , and we discuss strategies to further improve this figure of merit. @ 2015 AIP Publishing LLC. [http://dx.doi.org/10.1063/1.4932574]
\end{abstract}

Photonic wire antennas have recently emerged as a powerful solution to shape the emission of a point-like emitter into a directive Gaussian free-space beam. The integration of an isolated quantum dot (QD) in such structures opens appealing prospects for solid-state quantum optics, in particular, for the generation of non-classical states of light. Beyond the initial demonstration of bright single-photon sources, ${ }^{1-3}$ the broad operation bandwidth of these antennas is also a key asset to realize tunable single-photon sources ${ }^{4}$ or bright sources of entangled photon pairs. ${ }^{5}$ Specifically, nanowire antennas exploit the efficient spontaneous emission (SE) control provided by a single-mode high-index nanowire waveguide, whose far-field emission is tailored by a top taper. $^{6}$ The taper can either take the shape of a sharp needle or the one of a "photonic trumpet," So far, appreciable beam directivity has been demonstrated with both approaches, but collection optics with a numerical aperture (NA) that exceeds 0.7 are still mandatory to fully intercept the antenna output beam. ${ }^{1,3,4,7}$

Scaling up the dimensions of a photonic trumpet in order to enlarge its top facet represents a natural strategy to improve the output beam directivity. In this work, we first examine theoretically the relevance of this approach. We then report the realization of "giant" photonic trumpets with a $5 \mu \mathrm{m}$ wide top facet. Using Fourier microscopy, we map their far-field emission and show that $95 \%$ of the emitted beam is intercepted by a numerical aperture of 0.35 . Moreover, our measurements reveal a highly Gaussian angular profile, in agreement with the predicted overlap to a Gaussian beam $\mathcal{M}_{g}=0.98$. Extending the top facet also dramatically increases the overlap to the mode supported by a

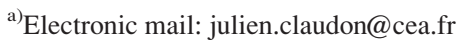

standard single-mode fiber (SMF), enabling an efficient direct coupling. The calculated transmission from the taper base to a cleaved fiber already reaches 0.59 , and we discuss future improvement directions.

The structure under investigation is made of a dielectric material with a large refractive index $\left(n_{t}=3.48\right)$, in a low index environment $(n=1)$. As illustrated in Fig. 1(a), its bottom section features a diameter $d_{b}=230 \mathrm{~nm}$, which ensures single-mode operation $\left(\mathrm{HE}_{11}\right)$ for a free-space operation wavelength $\lambda=925 \mathrm{~nm}$. Moreover, this diameter choice ensures an optimal control over the SE of an embedded
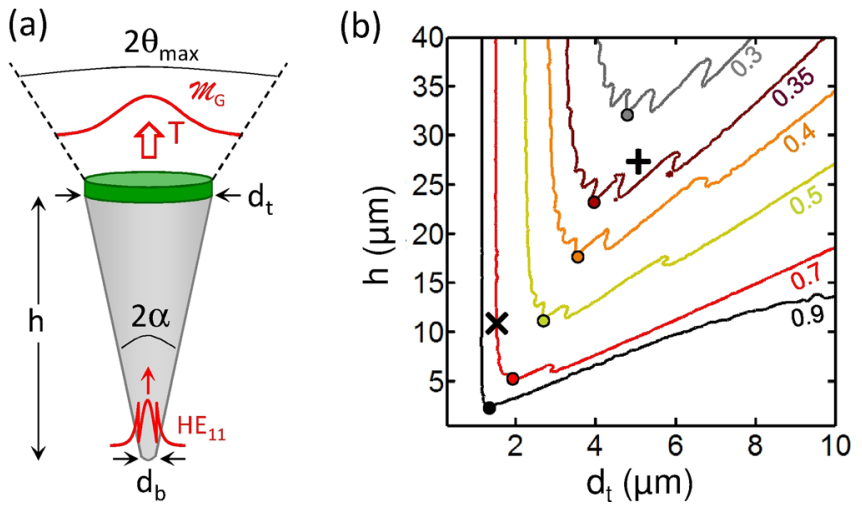

FIG. 1. (a) Geometry of the trumpet taper. Light is launched upward in the fundamental guided mode $\left(\mathrm{HE}_{11}\right)$ at the taper base. $T$ is the total transmission to a collection lens with a numerical aperture $\mathrm{NA}=\sin \left(\theta_{\max }\right)$, and $\mathcal{M}_{g}$ is the far-field overlap to a Gaussian beam. (b) Calculated transmission to a Gaussian beam $T_{g}=\mathcal{M}_{g} T$ in the $\left(d_{t}, h\right)$ plane for $d_{b}=230 \mathrm{~nm}$ and $\lambda=925$ $\mathrm{nm}$. Each contour line corresponds to $T_{g}=0.9$ for a given collection NA; the solid circles highlight the minimal taper dimensions compatible with those requirements. The cross corresponds to the taper described in Ref. 3, and the plus sign to this work. 
point-like emitter. ${ }^{8}$ Indeed, for an on-axis optical dipole, 95\% of the SE is funneled into the $\mathrm{HE}_{11}$ modes, with symmetric contributions to the modes propagating upward and downward. Moving up along the structure, the wire diameter increases linearly (total opening angle $2 \alpha$ ) to induce a lateral expansion of the guided mode. The top facet is located at a distance $h$ from the base and features a diameter $d_{t}$. It is covered with a $\lambda / 4$ anti-reflection coating which is composed of $\mathrm{Si}_{3} \mathrm{~N}_{4}$ (refractive index $n_{\text {ar }}=1.99$ ).

Qualitatively, the criterion for an adiabatic expansion of $\mathrm{HE}_{11}$ is determined from a comparison between $\alpha_{0}$, the diffraction angle of the mode inside the taper, and the sidewall tapering angle $\alpha$ (Ref. 9). $\alpha_{0}=\lambda /\left(\pi n_{\text {eff }} w_{0}\right)$, where $n_{\text {eff }}$ and $w_{0}$ are the effective index and waist ${ }^{10}$ of $\mathrm{HE}_{11}$. For the high contrast waveguide investigated here, as soon as the local diameter $d$ exceeds $0.5 \mu \mathrm{m}, n_{\text {eff }} \approx n_{t}$ and $w_{0} \approx 0.75(d / 2)$. Adiabatic transmission of $\mathrm{HE}_{11}$ is achieved when $\alpha \ll \alpha_{0}$. Larger taper angles, on the order of $\alpha_{0}$, result in the onset of conversion to higher order modes, with oscillations arising from interference effects. Finally, for $\alpha \gg \alpha_{0}$, one enters a deeply multimode regime: a large fraction of the electromagnetic energy is irreversibly transferred to higher order guided modes. At the taper base, $\alpha_{0}$ largely exceeds $\alpha$. Moving up the taper, $\alpha_{0}$ decreases as $d^{-1}$ to reach a minimum at the top facet level. The breakdown of adiabatic propagation occurs at a typical height $h_{0}=\lambda /\left(0.75 \pi n_{t} \alpha^{2}\right)$ which corresponds to $\alpha_{0}=\alpha$. If $h \lesssim h_{0}$, the whole taper operates in the adiabatic regime, yielding large modal transmission for $\mathrm{HE}_{11}$. For larger tapers, light propagation involves several guided modes above $h_{0}$.

We now focus on the taper output beam. In the following theoretical analysis, light is launched at the taper base into the upward $\mathrm{HE}_{11}$ mode, propagates along the taper, and is collected in free-space by an ideal thin lens with a NA. The taper transmission to a Gaussian beam can be decomposed as $T_{g}=\mathcal{M}_{g} T$, where $T$ is the total transmission into the lens, and $\mathcal{M}_{g}$ is the overlap between the output beam and a Gaussian beam. As detailed in the supplementary material of Ref. 3 (see also Ref. 11), $T_{g}$ is calculated by optimizing both the waist dimension of the target Gaussian beam and the location of the lens focal plane.

Figure 1(b) shows contour lines corresponding to $T_{g}=$ 0.9 in the $\left(d_{t}, h\right)$ plane for various values of the NA of the collection optics. For an adiabatic taper, the output beam is solely defined by the diffraction of the $\mathrm{HE}_{11}$ mode at the top facet level. For relevant geometries $\left(d_{t}>1 \mu \mathrm{m}\right)$, the spatial profile of this mode is very close to a Gaussian. The location of the lens focal plane that optimizes $T_{g}$ coincides with the top facet, and one obtains large $T_{g}$ into adequate collection NAs. This regime is revealed by the independence on $h_{t}$ for small $d_{t}$ values (vertical part of contour lines in Fig. 1(b)).

In a non-adiabatic taper, $T_{g}$ depends both on $d_{t}$ and $h$, with oscillations associated with mode interferences. For a given $d_{t}$, a multimode taper generally features a larger beam divergence than a single-mode taper. However, and quite surprisingly, a satisfying Gaussian output beam can still be obtained for significantly multimode tapers if the focal plane is set to a distance $\sim\left(h-h_{0}\right) / n_{t}$ below the top facet. This suggests that when adiabatic transmission breaks down, the mode diffracts as in the bulk material, with small deviations associated with the finite set of available guided modes. In this simple picture, the taper upper part does not lead to further improvements of the output beam directivity.

For each NA, one can define minimal taper dimensions $\left(d_{t, m}, h_{m}\right)$ ensuring $T_{g}=0.9$. Such a minimal geometry is identified by solid circles in the figure and is found close to the single-mode regime. The parabolic dependence of $h_{m}$ on $d_{t, m}$ is reminiscent of the condition $\alpha \sim \alpha_{0}$ discussed above. For NA $=0.7$, a very compact structure, with $h_{m}=5 \mu \mathrm{m}$, is sufficient to ensure $T_{g}=0.9$. However, further improvements of the beam directivity become increasingly demanding in terms of taper height. As an example, $h_{m}$ should be increased up to $24 \mu \mathrm{m}$ in order to obtain the same transmission into a NA of 0.35 .

In the second part of the paper, we demonstrate the fabrication of such "giant" photonic trumpets and measure their far-field emission properties. Figure 2(a) is a scanning electron microscope image of a $26.8 \mu \mathrm{m}$ high photonic trumpet that is supported by a pyramidal pedestal. At the connection with the pedestal, the waveguide supports a single guided mode $\left(d_{b}=210 \mathrm{~nm}\right)$. The total tapering angle is $2 \alpha=10.5^{\circ}$ and the top diameter $d_{t}=5.15 \mu \mathrm{m}$. For optical characterization, the device embeds self-assembled InAs QDs as an internal light source. The QDs are located $\sim 150 \mathrm{~nm}$ below the connection with the pedestal. The structure is mostly composed of $\mathrm{Al}_{0.05} \mathrm{Ga}_{0.95} \mathrm{As}$, except the dot section for which GaAs is used as barrier material. Compared to Ref. 3, the taper height and top diameter have been multiplied by more than a factor of 2 and 3, respectively. In brief, fabrication starts with the growth of a planar sample by molecular beam epitaxy. A hard etching mask is then defined using e-beam lithography, nickel deposition, and lift-off. The trumpets are etched in a reactive ion etching chamber, using a $\mathrm{SiCl}_{4}-\mathrm{Ar}$ gas chemistry. Finally, the remaining Ni mask is removed using wet chemistry.

Optical characterization is performed in a cryogenic microphotoluminescence ( $\mu \mathrm{PL}$ ) setup. The sample is kept at
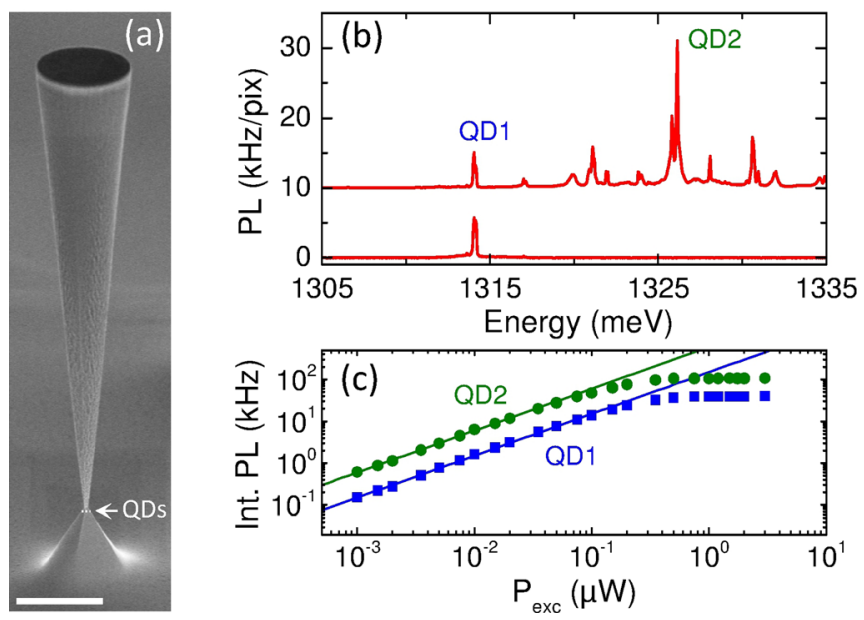

FIG. 2. Sample and microphotoluminescence spectra. (a) Scanning electron microscope image of the sample (tilted view). The horizontal scale bar represents $5 \mu \mathrm{m}$. (b) Top trace: Microphotoluminescence spectrum, acquired under pulsed excitation with a mean power $P_{\mathrm{ex}}=200 \mathrm{nW}$ (vertically offset for clarity). Bottom trace: Isolation of a single QD line using additional interferometric filters. (c) Spectrally integrated CCD counts versus $P_{\mathrm{ex}}$ for QD lines 1 and 2. The solid lines show linear dependencies. 
liquid helium temperature $(\sim 4 \mathrm{~K})$ in a cold finger cryostat. The QD luminescence is excited by a pulsed laser beam (repetition rate $76 \mathrm{MHz}$ ) focused on the sample with a microscope objective $(\mathrm{NA}=0.6)$. The excitation energy $\left(E_{\mathrm{ex}}=1.433 \mathrm{eV}\right)$ is tuned below the GaAs and $\mathrm{Al}_{0.05} \mathrm{Ga}_{0.95} \mathrm{As}$ band gaps, in the absorption continuum of the QD's wetting layer. The same objective collects the QD luminescence, and a low pass filter blocks stray laser light. For spectral analysis, the luminescence signal is directed towards a grating spectrometer equipped with a CCD camera.

A $\mu$ PL spectrum is shown in Fig. 2(b) (top trace): it is composed of sharp lines, associated with the recombination of excitonic complexes trapped in individual QDs. At saturation, the brightest lines (for example, QD2) typically feature a spectrally integrated CCD count rate of $\sim 100 \mathrm{kHz}$ [Fig. 2(c)], which corresponds to a top extraction efficiency $\epsilon_{\uparrow} \sim 0.1$. The isolated line QD1, that will be investigated latter, features a twice lower $\epsilon_{\uparrow}$. In the open waveguide (no bottom mirror) investigated here, the theoretical maximum for $\epsilon_{\uparrow}$ is 0.47 . It would be achieved for a QD with a stable charge state, located on the waveguide axis, above the connection with the pedestal. We stress that this reduced $\epsilon_{\uparrow}$ is not a concern for the main goal of this work, namely, the investigation of the taper far-field emission using Fourier microscopy.,12

As illustrated in Fig. 3(a), the taper emission can be decomposed on a set of plane waves which leave the top facet with a direction defined by the polar and azimuthal angles $\theta$ and $\varphi$. These plane waves are focused on a point $(\rho(\theta), \varphi)$ of the objective back focal plane. After calibration of the objective response (function $\rho(\theta)$ and transmitted intensity versus $\theta$ ), we image this plane with a CCD camera which yields the far-field intensity per unit solid angle $\frac{\mathrm{d} I}{\mathrm{~d} \Omega}(\theta, \varphi)$. The objective calibration is detailed in the supplementary material. ${ }^{13}$ To a very good approximation, the function $\rho(\theta)$ is linear. The maximum imaging angle $\theta_{\max }=37^{\circ}$ is limited by the objective NA; this limit appears as a solid circle in the far-field map presented in Fig. 3. Close to the angle cut-off, the objective transmission has dropped by
$25 \%$ relative to its on-axis value. The data presented in the following are corrected from the objective response. To validate the setup, we have also imaged the far-field emission of a single InAs QD buried in an unprocessed GaAs planar sample. The resulting broad far-field emission pattern matches very well the theoretical predictions (see supplementary material ${ }^{13}$ ).

We perform angle-resolved far-field measurements on a single QD embedded in the trumpet. We focus on the spectrally isolated line QD1, which can be filtered using a combination of bandpass interferometric filters [bottom trace in Fig. 2(b)]. As shown in Fig. 3(b), the trumpet under study emits a very directive beam which is fully intercepted by the objective. Moreover, as shown in Fig. 3(c), far-field measurements conducted on the QD ensemble yield an identical pattern, which is a direct proof of the single-mode nature of the bottom part of the waveguide. For quantitative analysis, we estimate the emission intensity intercepted within a cone of opening angle $\theta, I_{\text {int }}(\theta)=\int_{0}^{2 \pi} \mathrm{d} \varphi \int_{0}^{\theta} \frac{\mathrm{d} I}{\mathrm{~d} \Omega}(\theta, \varphi) \sin \theta \mathrm{d} \theta$. Since the beam is fully intercepted by the objective, the transmission to an optics with a collection angle $\theta$ is given by $T=I_{\text {int }}(\theta) / I_{\text {int }}\left(\theta_{\max }\right)$. As demonstrated in Fig. 3(d), a modest $0.35 \mathrm{NA}$ is sufficient to collect $95 \%$ of the taper output beam. This represents a significant improvement over previously reported nanowire antennas (needle- or trumpetlike): up to now, these required NAs larger than 0.7 for efficient light collection. ${ }^{1,3,4,7}$

Furthermore, the cuts along $\varphi=0^{\circ}$ and $\varphi=90^{\circ}$, which are presented in the top and right panels in Fig. 3(b), reveal highly Gaussian beam profiles. Both traces can be fitted to the same function $\propto \exp \left[-2\left(\theta / \theta_{0}\right)^{2}\right]$, yielding a beam divergence angle $\theta_{0}=16^{\circ}$. Except for acquisition noise, the data do not show any deviation from the Gaussian law. This confirms simulation results, which predict an overlap to a Gaussian beam $\mathcal{M}_{g}=0.98$ for a 0.35 collection NA. Although the Gaussian character of the beam emitted by needle nanowires has recently been demonstrated ${ }^{7}$ and was predicted for trumpet tapers, ${ }^{3}$ we stress that the present

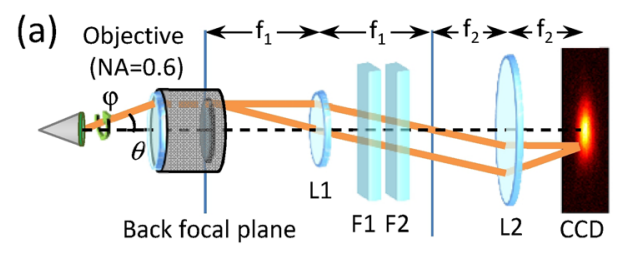

(d) Collected fraction of the output beam

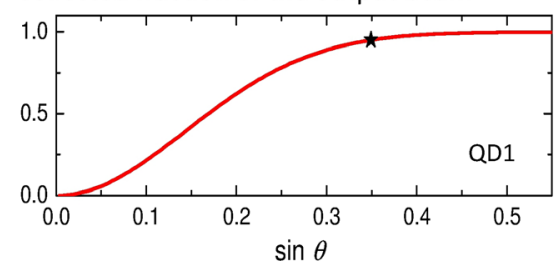

(b) QD1 (filters F1 and F2)

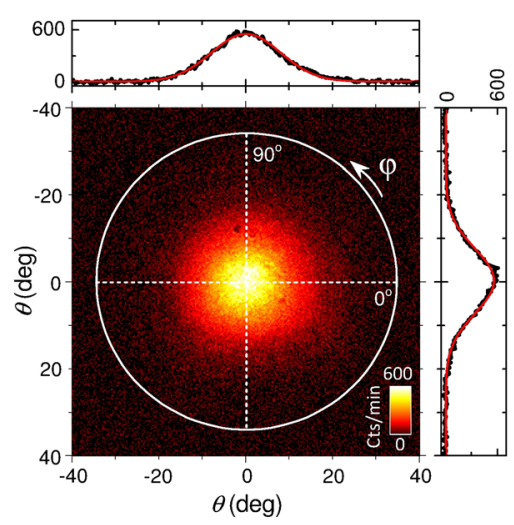

\section{(c) All QDs (no filtering)}

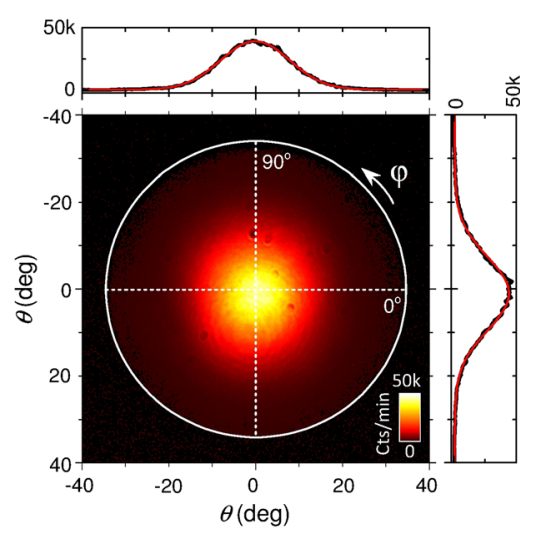

FIG. 3. Far-field imaging. (a) Fourier microscopy setup. L1 and L2 are lenses of focal length $f_{1}=40 \mathrm{~cm}$ and $f_{2}=20 \mathrm{~cm}$; F1 and F2 are bandpass interferometric filters. (b) Far-field intensity map in the $(\theta, \varphi)$ plane, acquired on the spectrally filtered line QD1 shown in Fig. 2(b). The solid circle corresponds to the maximum collection angle of the microscope objective $\left(\theta_{\max }=37^{\circ}\right)$. The dark CCD counts have been subtracted, and the data are corrected for the objective response. The upper panel is a profile measured along $\varphi=0^{\circ}$, and the right one is a profile measured along $\varphi=90^{\circ}$ ("lateral" integration over 5 CCD pixels). The solid lines are fit to a Gaussian profile, yielding identical beam diffraction angle $\theta_{0}=16^{\circ}$ for both profiles. (c) Same measurement as in (b), without spectral filtering. All QDs contribute to the far-field map. (d) Collected fraction of the beam intensity versus the collection NA, determined from partial angular integration of the measurements shown in (b). The star marks a collected fraction of $95 \%$. 
nanowire antenna offers an unprecedented combination of directive and Gaussian emission. Beyond nanowire photonics, similar far-field characteristics are only offered by state-of-the-art micropillar cavities. ${ }^{14,15}$ These features simplify the efficient out-coupling to a single-mode fiber using free-space coupling optics, and, reversibly, the incoupling of an external Gaussian laser beam. In analogy with a symmetric micropillar cavity that embeds a QD, ${ }^{16}$ the structure investigated in this work is particularly well suited to explore optical nonlinearities at the single-photon level. Interestingly, the broadband nature of a waveguide approach enables multicolor nonlinearities based on complex emitter level schemes. ${ }^{17}$

The "butt" coupling to a SMF constitutes another appealing perspective of this work. ${ }^{18}$ In particular, such a device would result in a practical, alignment-free source for the long-distance distribution of quantum light. As illustrated in Fig. 4(a), we first consider the coupling to the cleaved facet of a standard SMF $(\mathrm{NA} \approx 0.1)$. Such a coupling has been recently demonstrated, ${ }^{19}$ using the first generation of trumpets presented in Ref. 3. In the following theoretical analysis, a perfectly matched anti-reflection layer with $n_{\mathrm{ar}}=2.25$ is inserted between the trumpet and the fiber cleaved facet. For a taper with a single-mode base, the transmission from the taper base to the fiber reads $T_{f}=T_{\mathrm{HE}_{11}} \times T_{\text {facet }}$, where $T_{\mathrm{HE}_{11}}$ is the $\mathrm{HE}_{11}$ modal transmission of the taper, and $T_{\text {facet }}$ is the top facet to fiber modal transmission. The taper described in Ref. 3 offers an excellent $T_{\mathrm{HE}_{11}}=0.99$. However, the modest top facet diameter $\left(d_{t}=1.6 \mu \mathrm{m}\right)$ leads to a poor overlap to the fiber mode and limits $T_{\text {facet }}$ to 0.11 [cross in Fig. 4(b)]. The "giant" trumpet demonstrated in this work features a much larger $T_{\text {facet }}=0.74$ [plus sign in the figure]. Despite a slightly smaller $T_{\mathrm{HE}_{11}}=0.8, T_{f}$ is increased by a factor of 5 and reaches 0.59 . Going beyond this result will require the simultaneous optimization of $T_{\mathrm{HE}_{11}}$ and $T_{\text {facet }}$. An optimal $T_{\text {facet }}=0.95$ is achieved for $d_{t}=8.6 \mu \mathrm{m}$. As shown in Fig. 4(c), a linear taper with this $d_{t}$ should feature a height $h>$ $118 \mu \mathrm{m}$ to ensure $T_{\mathrm{HE}_{11}}>0.90$. Such structures could be defined in a thinned GaAs wafer, after a flip-chip step, and using a robust hard mask which can sustain very deep etching. Alternatively, an additional tapering of the fiber ${ }^{20}$ allows


FIG. 4. (a) "Butt" coupling to the cleaved facet of a standard single-mode fiber. Fiber characteristics: core and cladding indices: 1.4574 and 1.4516; core diameter: $d_{\text {core }}=4.3 \mu \mathrm{m}$. (b) The plot shows the calculation of $T_{\text {facet }}$, the facet to facet modal transmission as a function of $d_{t}$, the trumpet top facet diameter. The cross corresponds to the taper described in Ref. 3, and the plus sign to this work. (c) Minimal taper height leading to a taper modal transmission $T_{\mathrm{HE}_{11}}>0.9$, as a function of $d_{t}$. reducing the fiber mode size down to the micron range, thus decreasing a lot the constraints on $d_{t}$ and $h$.

To conclude, we have demonstrated "giant" photonic trumpets which funnel the emission of a single QD into a very directive and Gaussian output beam. Beyond free-space quantum optics experiments, these structures can also be directly coupled with high efficiency to a cleaved singlemode fiber. Envisioned applications include practical sources of quantum light, as well as recently proposed near-field QD-electrical field sensors and single-plasmon launchers. ${ }^{19}$

CEA authors acknowledge the support of the European Union Seventh Framework Program 209 (FP7/2007-2013) under Grant Agreement No. 601126210 (HANAS). CEA authors, N.G. and J.M. acknowledge the support of the European Metrology Research Programme [project SIQUTE (Contract No. EXL02)]. M.R. acknowledges the support of the European Research Council (STG 25868). M.M. and J.T. acknowledge funding from the Swiss National Centre of Competence in Research "QSIT-Quantum Science and Technology." Sample fabrication was carried out in the "Plateforme Technologique Amont (PTA)" and CEA LETI MINATEC/DOPT clean rooms.

${ }^{1}$ J. Claudon, J. Bleuse, N. S. Malik, M. Bazin, P. Jaffrennou, N. Gregersen, C. Sauvan, P. Lalanne, and J.-M. Gérard, Nat. Photonics 4, 174 (2010).

${ }^{2}$ M. E. Reimer, G. Bulgarini, N. Akopian, M. Hocevar, M. B. Bavinck, M. A. Verheijen, E. P. A. M. Bakkers, L. P. Kouwenhoven, and V. Zwiller, Nat. Commun. 3, 737 (2012).

${ }^{3}$ M. Munsch, N. S. Malik, J. Bleuse, E. Dupuy, A. Delga, J.-M. Gérard, J. Claudon, N. Gregersen, and J. Mørk, Phys. Rev. Lett. 110, 177402 (2013).

${ }^{4}$ P. E. Kremer, A. C. Dada, P. Kumar, Y. Ma, S. Kumar, E. Clarke, and B. D. Gerardot, Phys. Rev. B 90, 201408 (2014).

${ }^{5}$ M. A. M. Versteegh, M. E. Reimer, K. D. Joens, D. Dalacu, P. J. Poole, A. Gulinatti, A. Giudice, and V. Zwiller, Nat. Commun. 5, 5298 (2014).

${ }^{6}$ J. Claudon, N. Gregersen, P. Lalanne, and J.-M. Gérard, ChemPhysChem 14, 2393 (2013).

${ }^{7}$ G. Bulgarini, M. E. Reimer, M. B. Bavinck, K. D. Jöns, D. Dalacu, P. J. Poole, E. P. A. M. Bakkers, and V. Zwiller, Nano Lett. 14, 4102 (2014).

${ }^{8}$ J. Bleuse, J. Claudon, M. Creasey, N. S. Malik, J.-M. Gérard, I. Maksymov, J.-P. Hugonin, and P. Lalanne, Phys. Rev. Lett. 106, 103601 (2011).

${ }^{9}$ A. F. Milton and W. K. Burns, IEEE J. Quantum Electron. 13, 828 (1977).

${ }^{10} w_{0}$ is defined as the radial distance for which the amplitude of the dominant electric field component is divided by $e$.

${ }^{11}$ M. Munsch, N. S. Malik, J. Bleuse, E. Dupuy, A. Delga, J.-M. Gérard, J. Claudon, N. Gregersen, and J. Mørk, Phys. Rev. Lett. 111, 239902 (2013).

${ }^{12}$ G. Grzela, R. Paniagua-Domínguez, T. Barten, Y. Fontana, J. A. SánchezGil, and J. Gómez Rivas, Nano Lett. 12, 5481 (2012).

${ }^{13}$ See supplementary material at http://dx.doi.org/10.1063/1.4932574 for (i) the calibration of the microscope objective response and (ii) the far-field emission of an InAs QD embedded in an unprocessed GaAs planar sample.

${ }^{14}$ S. Strauf, N. G. Stoltz, M. T. Rakher, L. A. Coldren, P. M. Petroff, and D. Bouwmeester, Nat. Photonics 1, 704 (2007).

${ }^{15}$ O. Gazzano, S. Michaelis de Vasconcellos, C. Arnold, A. Nowak, E. Galopin, I. Sagnes, L. Lanco, A. Lemaitre, and P. Senellart, Nat. Commun. 4, 1425 (2013).

${ }^{16}$ V. Loo, C. Arnold, O. Gazzano, A. Lemaitre, I. Sagnes, O. Krebs, P. Voisin, P. Senellart, and L. Lanco, Phys. Rev. Lett. 109, 166806 (2012).

${ }^{17}$ P. Kolchin, R. F. Oulton, and X. Zhang, Phys. Rev. Lett. 106, 113601 (2011).

${ }^{18}$ F. Haupt, S. S. R. Oemrawsingh, S. M. Thon, H. Kim, D. Kleckner, D. Ding, D. J. Suntrup III, P. M. Petroff, and D. Bouwmeester, Appl. Phys. Lett. 97, 131113 (2010).

${ }^{19}$ D. Cadeddu, J. Teissier, F. Braakman, N. Gregersen, P. Stepanov, J.-M. Gérard, J. Claudon, R. J. Warburton, M. Poggio, and M. Munsch, e-print arXiv: 1506.06635 .

${ }^{20}$ L. Ding, C. Belacel, S. Ducci, G. Leo, and I. Favero, Appl. Opt. 49, 2441 (2010). 